\title{
Identidad, organización y estrategia en dos movimientos que pugnan por la soberanía alimentaria en México
}

\author{
JOALINÉ PARDO NÚÑEZ
}

\begin{abstract}
Analizo y comparo dos movimientos por la soberanía alimentaria en México a partir de las identidades colectivas que integran cada uno, las formas en que se organizan para trabajar y decidir, así como las decisiones estratégicas que toman para orientar su acción y movilizarse. Ambos contribuyen al debate por la construcción de un nuevo proyecto alimentario en México y estudian problemas similares desde perspectivas diferentes para revisar el papel del campo. Los actores colectivos de cada caso comparten un pasado común anclado en el movimiento campesino, pero se reconstituyen como dos proyectos separados a partir de evaluaciones diferenciadas de la acción y sus resultados, para dar lugar a dos movimientos distintos en su configuración, organización y postura ante el Estado.
\end{abstract}

PALABRAS CLAVE: Soberanía alimentaria, arenas, demandas, tácticas, identidades

Identity, Organization and Strategy in Two Movements Struggling for Food Sovereignty in Mexico

I analyze and compare two movements for food sovereignty in Mexico, taking into account the collective identities that constitute each movement, the organizational forms they use for working and deciding, as well as the strategic decisions they make to orient their action. Both movements contribute to the debate for the construction of a new agrifood project in Mexico, addressing from different views similar problems to tackle the role of the countryside. Collective actors in each case share a common past in the campesino movement, but they constituted two projects, deriving from differentiated evaluations of the action and its results, that turn out as two different movements in their social configuration, their organization and its position towards the State. 


\section{Introducción}

a distinción entre comunidades indígenas que confluyen en torno a una

identidad étnica, culturalmente diferenciada, geográficamente situada y de adscripción involuntaria, y organizaciones campesinas construidas socialmente a partir de afinidades por actividad productiva, con independencia de rasgos geográficos o culturales, no había sido relevante para un movimiento campesino en México, pues desde mediados del siglo XX se luchaba por la tenencia de la tierra (1970-1976), por subsidios al campo en un segundo periodo (1977-1983) y por tener incidencia en la política agrícola, en un tercero (19842000) (Rubio, 2003; 2006).

A partir de los sucesos de un nuevo momento de movilizaciones respecto a la soberanía alimentaria y la evaluación de los resultados de la acción, la identidad en relación con la pertenencia étnica o con intereses productivos se hizo patente y llevó a que los participantes de un movimiento, hasta entonces unificado, se separaran para confrontar al Estado desde sus afinidades ideológicas y definieran estrategias específicas con base en marcos de interpretación compartidos.

En la actualidad, los dos movimientos resultantes de dicha separación ponen al maíz en el centro de sus demandas, con el concepto de soberanía alimentaria como marco:

La soberanía alimentaria es el derecho de los pueblos a alimentos nutritivos y culturalmente adecuados, accesibles, producidos de forma sostenible y ecológica, y su derecho a decidir su propio sistema alimentario y productivo [...], por encima de las exigencias de los mercados y de las empresas (Nyéléni Newsletter, 2007).

La detección de polen de maíz transgénico y la liberación de aranceles para productos básicos en el Tratado de Libre Comercio de América del Norte (TLCAN) obligaron a cada colectivo participante en el movimiento a tomar decisiones de 
fondo y forma para guiar su lucha subsecuente y analizar el concepto de soberanía alimentaria a partir de realidades diversas. Evaluaciones diferenciadas de la acción y sus resultados llevaron a redefinir adscripciones identitarias y formas organizativas. En la actualidad, con la demanda principal de soberanía alimentaria, la Red en Defensa del Maíz, un movimiento indígena, y la Campaña Nacional Sin Maíz No Hay País, de carácter campesino, son los dos movimientos que enarbolan elementos culturales, económicos y políticos distintos, y se relacionan con el Estado de manera diferenciada.

Ambos se oponen al régimen alimentario neoliberal, es decir, al creciente favoritismo del Estado hacia corporativos y asociaciones transnacionales para controlar los insumos y cadenas de producción agrícola. ${ }^{1}$ Sin embargo, las formas de organización interna, así como las estrategias que emplean para confrontar al régimen, difieren en cuanto a los actores que se consideran centrales en cada movimiento y el papel que se otorga al Estado.

El presente análisis de las diferencias entre ambos movimientos se basa en tres elementos: identidad, organización y decisiones estratégicas. El punto de partida es pensar que la identidad de cada actor colectivo conlleva filtros ideológicos y normas de conducta que posibilitan o restringen la asociación con otros (Kurtz, 2002), que definen poco a poco relaciones y posturas ante el Estado, actores del ámbito político y otros actores de la sociedad civil.

La información que aquí se presenta es resultado de un año y medio de participación en ambos movimientos (2010-2011), como parte de la investigación para la tesis doctoral. Durante ese periodo, se llevó a cabo la documentación etnográfica a partir de la observación participativa y de una serie de entrevistas abiertas a 15 líderes o representantes de organizaciones de la Campaña Nacional Sin Maíz No Hay País y a 20 personas reconocidas como autoridades de la Red en Defensa del Maíz. La información fue sistematizada y analizada por categorías relacionadas con momentos clave del movimiento, percepción de roles y funciones de los actores del movimiento y del Estado, así como decisiones estratégicas. Esto permitió dar testimonio de los motivos de separación del movimiento, las características actuales de cada colectivo y las particularidades que impiden la reunificación.

\section{Identidad y estrategia}

La identidad social de un grupo es el proceso de equiparación y reconocimiento que cohesiona a sus integrantes en torno a características particulares, culturalmente valoradas como propias y únicas (Cardoso, 2007: 54). También implica la valoración y separación de "otros", y el posicionamiento de estas características en las relaciones intergrupales (Lehman, 1967: 105-107). La identidad permite crear marcos de significado para entender y problematizar la realidad (Kane, 1997; Freeman, 1999). Estos marcos constituyen esquemas de interpretación que facilitan localizar, percibir, identificar y etiquetar situaciones; sirven para organizar la experiencia y orientar la acción, individual y colectiva, al dotar de sentido los acontecimientos (Snow y Benford, 1988: 201). También hacen posible la construcción de evaluaciones para la acción social

Según McMichael (2006), el actual régimen corporativo, desde 1980, emergió del shock económico mundial de la década de 1970 y los acuerdos internacionales neoliberales. Como tendencia, los gobiernos promovieron programas de ajustes estructurales con los que disminuyeron aranceles, eliminaron precios de garantía y redujeron subsidios a pequeños productores. Este régimen se caracteriza como un periodo hiperindustrial en el que se firmaron tratados regionales de libre comercio, se reguló la privatización de tierras y recursos naturales, hubo reducción gradual de subsidios a la agricultura, y se promovieron la agroindustria y los paquetes biotecnológicos. 


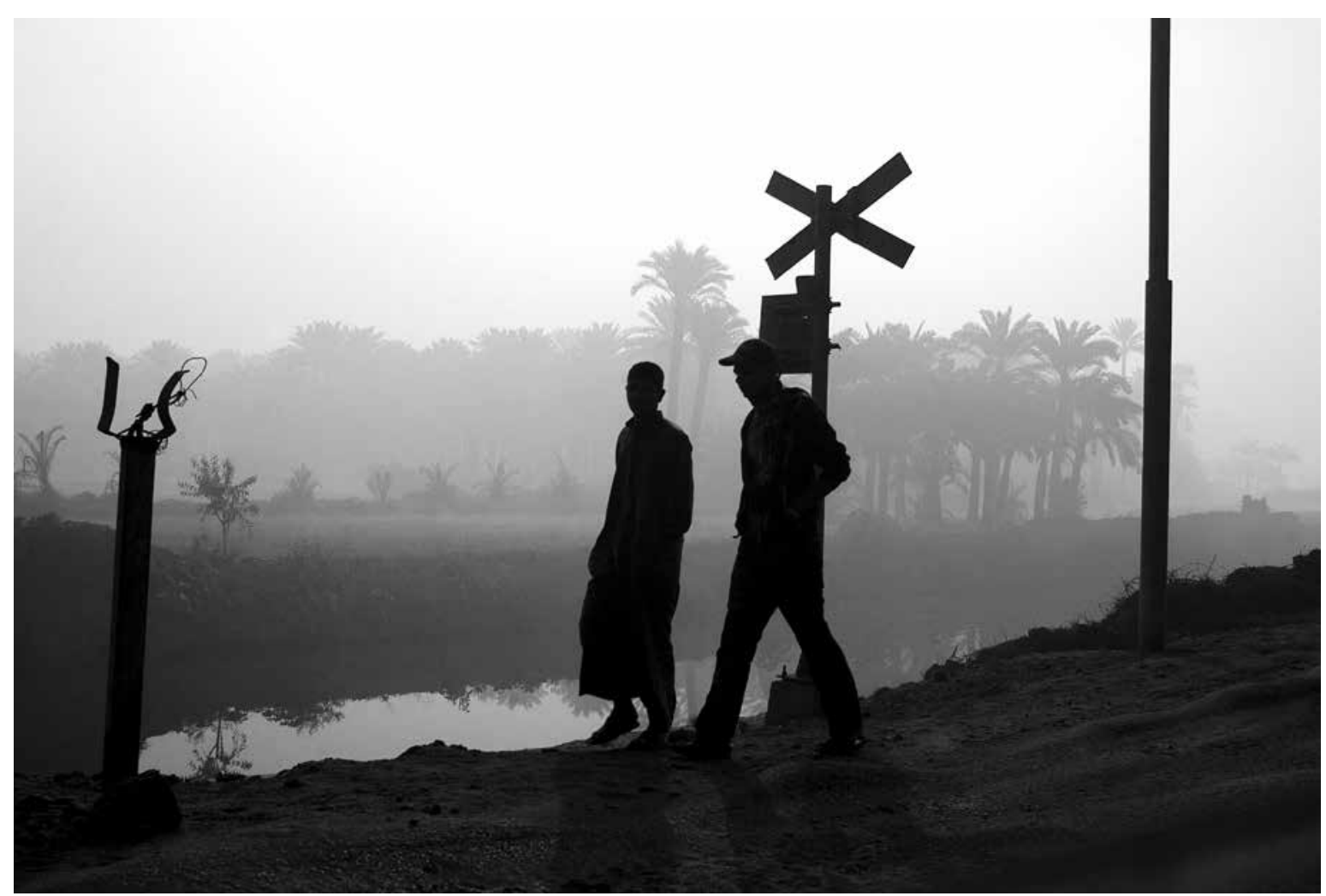

Ricardo Ramirez Arriola/360 • Camino hacia El Cairo, Egipto, 2008.

en función de lo que se considera adecuado o válido, según los valores y normas que acompañan la identidad (Polleta y Jasper, 2001; Meyer y Staggenborg, 2007). En los movimientos multiidentitarios, cada grupo se define en torno a elementos culturales distintos (Kurtz, 2002). Se entiende que no hay un "nosotros" colectivo, sino identidades que comparten representaciones de un problema, así como valores para confrontarlo (Polleta y Jasper, 2001).

Las identidades colectivas que convergen en ambos movimientos son múltiples: organizaciones campesinas que se agrupan en torno a intereses productivos —Campaña-, organizaciones y comunidades indígenas con intereses culturales y territoriales - Red-, así como organizaciones de la sociedad civil de índole ambiental, académico o de defensa de los derechos humanos. En un primer nivel organizativo, cada grupo participa con y basado en una identidad propia, con objetivos particulares y de acuerdo con su normatividad interna. En un segundo nivel, el movimiento aparece como instancia de deliberación y acción en el que prevalecen normas de funcionamiento y jerarquías acordadas entre los grupos del primer nivel.

\section{Decisiones estratégicas}

De acuerdo con Meyer y Staggenborg (2007), las acciones contenciosas que realiza un movimiento son producto de sus decisiones sobre arenas o 
ámbitos de incidencia, demandas o exigencias, y tácticas o acciones contenciosas. Una decisión se considera estratégica cuando define patrones generales de acción que caracterizan al movimiento de forma cultural, política y argumentativa (Staggenborg, 1998: 192); se toma en momentos considerados clave y da lugar a éxitos o fracasos en función de sus objetivos (Meyer y Staggenborg, 2007).

La estrategia refleja aspectos cruciales de un movimiento: sus formas de enmarcar o delimitar el problema, las formas aceptables para resolverlo y el ideal de sociedad que busca (Schelling, 1960, citado en Ganz, 2000).

Para profundizar en los elementos de decisión estratégica, debe tenerse en cuenta que:

1. Las arenas o ámbitos para la acción son los lugares en los que se colocan las demandas y se confronta a los adversarios. La selección de arenas refleja las actitudes contenciosas del movimiento, pues conlleva la evaluación de actores sociales y políticos que provocan emociones y reacciones. Apuntarlos implica validarlos como interlocutores legítimos (Smithey, 2002).

2. Las demandas constituyen exigencias de cambio que, de cumplirse, mejorarían la vida de los miembros del movimiento o de los sectores a los que pretende representar (Meyer y Staggenborg, 2007). Las demandas requieren consenso amplio entre los actores del movimiento (Lofland, 1996) y se escogen según la evaluación colectiva de los mejores marcos discursivos en un momento determinado (Kokogiannis, 2010: 7).

3. Las tácticas son las formas que toma la acción colectiva. Son críticas por los resultados que aspiran conseguir para la causa del movimiento (Edwards y McCarthy, 2004). Las tácticas dependen del repertorio de sus organizaciones y de la evaluación de experiencias anteriores del movimiento, que descarta las que no han sido exitosas (Freeman, 1999).

\section{La génesis de dos movimientos}

En 2001, la publicación de un artículo en la revista Nature, que mencionaba la detección de polen de maíz transgénico en milpas tradicionales (Quist y Chapela, 2001), condujo a una serie de organizaciones no gubernamentales (ONG) a convocar un foro para discutir las implicaciones de la detección de maíz transgénico en Oaxaca (Marielle y Peralta, 2007). Estas ONG, entre las que destaca el Centro de Estudios para el Cambio en el Campo Mexicano (Ceccam), habían colaborado con organizaciones campesinas independientes del movimiento campesino, como la Unión Regional de Organizaciones Regionales Campesinas Autónomas (Unorca), que también convocaron al foro. En este espacio convergieron científicos, ${ }^{2}$ secretarías de Estado, ${ }^{3} \mathrm{ONG}{ }^{4}$ comunidades y organizaciones indígenas, así como organizaciones campesinas ${ }^{5}$ que se conocían de momentos anteriores de acción en el movimiento campesino. En este foro se acordó la constitución de un observatorio sobre los transgénicos para monitorear su presencia y tratar de prohibir su introducción, ante la falta de información existente sobre sus impactos (Red en Defensa del Maíz, 2003). Sin embargo, en 2003 se produciría un siguiente corte de desgravación del TLCAN, firmado por el gobierno en 1994.

2 Cerca de 15 académicos que después conformarían la Unión de Científicos Comprometidos con la Sociedad.

3 Entre ellas, la Secretaría del Medio Ambiente, Recursos Naturales y Pesca y la Comisión Nacional para la Biodiversidad.

$4 \quad$ Me refiero a organizaciones de la sociedad civil que surgen de la libre voluntad de sus miembros, sin representarlos política o socialmente, y cuyos objetivos son mejorar aspectos de la vida de la sociedad en la que se insertan, mediante financiamientos de agencias externas (Canto, 2002).

5 Para más detalles sobre las organizaciones asistentes, véase<http://redendefensadelmaiz.net/documentos-de-lared/conclusiones-del-primer-seminario-en-defensa-delmaiz/\#\&panel1-2>. 
Para ese año de preveía una nueva etapa de ampliación de los cupos de maíz blanco sin aranceles, que mermaría la activación del abasto interno para el país (Concheiro, Tarrío y Grajales, 2007). Ante esto, 12 organizaciones campesinas nacionales decidieron emprender movilizaciones contra el nuevo momento del TLCAN y formaron una organización sombrilla con el nombre de Movimiento El Campo No Aguanta Más (MECNAM); por esta razón, abandonaron de forma momentánea el espacio de la Red. El sector dominante del MECNAM no era el de los campesinos más pobres ni el de los grupos étnicos, pero ambos estaban incluidos en las organizaciones amplias (Sánchez, 2004).

Las organizaciones del MECNAM elaboraron un pliego petitorio que incluía renegociar el capítulo agropecuario del TLCAN, mejorar los presupuestos asignados al campo, ampliar la base de programas agropecuarios y firmar los acuerdos de San Andrés en su formato original (Suárez, 2005). Al MECNAM se unieron, a último momento, organizaciones que no formaban parte de la coalición sombrilla, como la Confederación Nacional Campesina y el Congreso Agrario Permanente, organizaciones de corte oficialista. $^{6}$

El MECNAM no obtuvo el apoyo del Ejército Zapatista de Liberación Nacional (EZLN) ni del Consejo Nacional Indígena (CNI), ${ }^{7}$ a pesar de que lo había solicitado cuando incorporó las reivindicaciones del movimiento indígena en sus peticiones, ante la reciente promulgación de una versión distorsionada de los acuerdos de negociación entre el gobierno y el EZLN, compilados en la propuesta de Ley de Concordia y Pacificación en 1996 (Serna, Valero y Díaz, 2010; Grammont, Mackinlay y Stoller, 2009). La negativa del EZLN y del CNI, que personificaban el movimiento indígena, se basó en la participación de las organizaciones campesinas oficialistas, cuya presencia dejaba ver que la negociación terminaría en acuerdos clientelares, como aumentos de montos presupuestales, y no en reformas profundas para el campo (Serna, Valero y Díaz, 2010). El movimiento indígena, además, vio de manera negativa que las organizaciones campesinas hubieran dejado pasar siete años desde la firma del TLCAN para intentar renegociar un capítulo (Serna, Valero y Díaz, 2010). Esto se oponía a la emergencia inmediata del EZLN y la reactivación del movimiento indígena el primer día de vigencia del tratado, en 1994.

El resultado de las movilizaciones fue, en efecto, un periodo de negociación con el gobierno que terminó con la firma del Acuerdo Nacional para el Campo (ANC) en 2003, no exento de confrontaciones internas. Las organizaciones indígenas que participaron en ese momento del movimiento lo hicieron como bases de las organizaciones amplias campesinas. Sin embargo, entrevistas con personas del Centro Nacional de Ayuda a las Misiones Indígenas (Cenami, Ciudad de México, 29 de junio de 2010) y de la Coordinadora Nacional de Organizaciones Cafetaleras (CNOC, Ciudad de México, agosto de 2010) dejan ver la gran frustración que experimentaron al conocer el rechazo del movimiento indígena tanto al MECNAM como a los resultados de la negociación con el gobierno.

También en 2003 se celebró en Cancún la Quinta Conferencia Ministerial de la Organización Mundial del Comercio (OMC), que constituyó un escenario de lucha y resistencia contra el orden global para el movimiento altermundista. ${ }^{8} \mathrm{El}$

En tanto que tenían un vínculo importante con el partido en el poder y retribuían cuotas de apoyo con votos en época de elecciones (Mackinlay y Otero, 2004), es decir, lo opuesto a las organizaciones independientes que habían participado en el foro inicial contra los transgénicos.

7 Máximo órgano para la toma de decisiones del movimiento indígena en México.

8 Conjunción de movimientos internacionales, intersectoriales, que se oponen a las políticas de libre comercio que favorecen a las corporaciones transnacionales como actores primordiales del mercado, en detrimento de las economías locales y el desarrollo endógeno (Pleyers, 2010). 


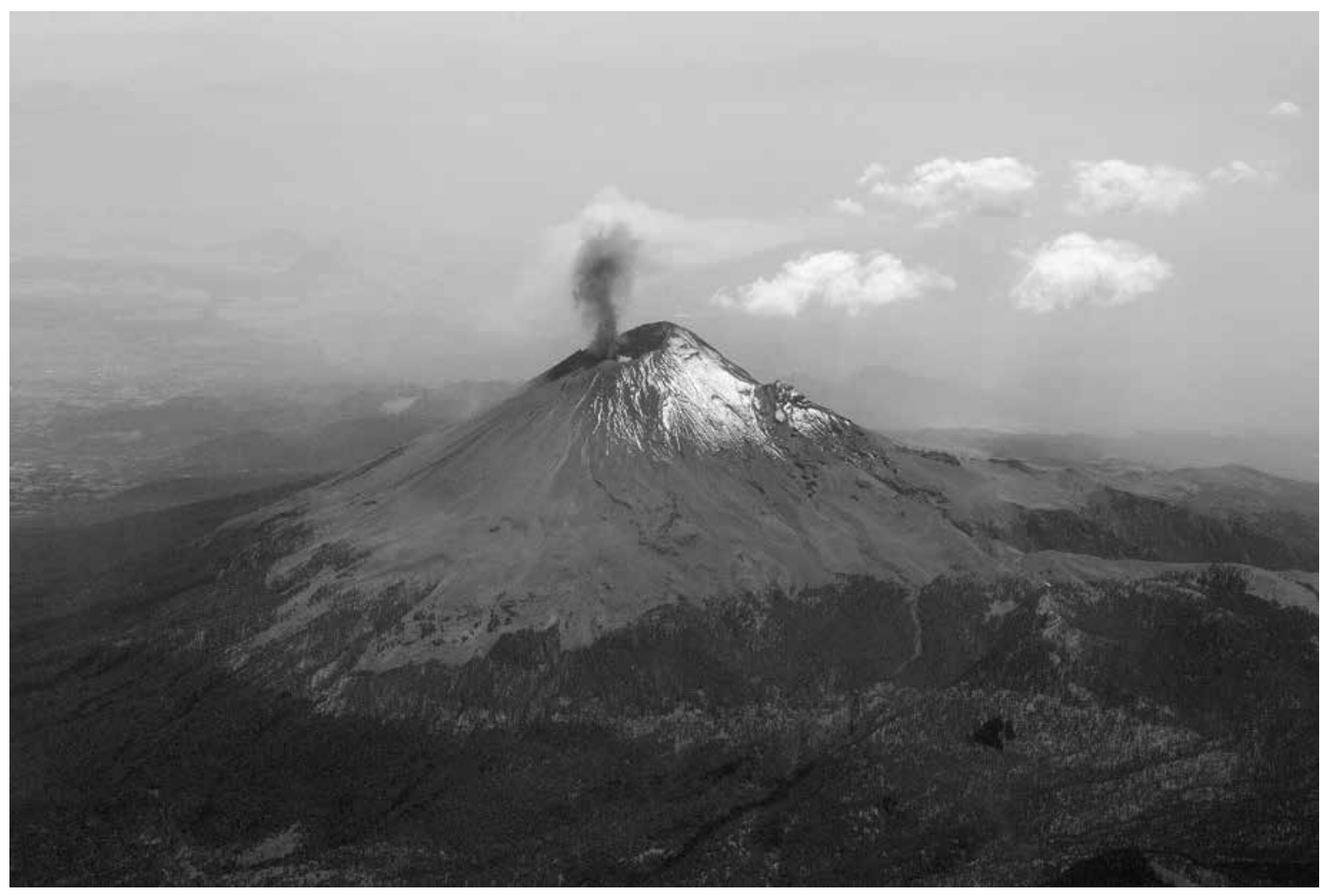

Prometeo Lucero • El volcán Popocatépetl emite una fumarola. Abril de 2015.

tema principal de esta ronda de negociaciones de la OMC era la agricultura, por ello la presencia del movimiento campesino nacional era indispensable (entrevista con integrante del CECCAM, Ciudad de México, 26 de agosto de 2010). Las diferencias entre las organizaciones a causa de la firma del ANC a principios de ese año tuvieron repercusiones en la asistencia a la protesta en Cancún. Mientras las organizaciones campesinas independientes firmantes del ANC $^{9}$ organizaron el Foro de los Pueblos, al que se sumaron la Red Mexicana de Acción contra el Libre Comercio y ONG internacionales, como Greenpeace y Oxfam — que además financió una parte importante de las actividades- (Pleyers, 2004), La Vía Campesina, como movimiento mundial, junto con organizaciones campesinas nacionales que se habían manifestado contra la firma del Acuerdo, convocaron al Foro Campesino e Indígena, que funcionó por separado. A éste llegó el mensaje grabado del EZLN en el que manifestaba su apoyo a la lucha de La Vía Campesina contra el orden neoliberal y la firma de acuerdos de libre comercio en materia agrícola y en biotecnología (De la Cueva, 2003). También llegaron representantes de pueblos indios integrantes del CNI. En este foro participaron ONG internacionales, aliadas estratégicas o asesores de algunas organizaciones campesinas, como

Asociación Mexicana de Uniones de Crédito del Sector Social (Amucss), Asociación Nacional de Empresas Comercializadoras de Productores del Campo (ANEC), CNOC, Frente Democrático Campesino (FDC), Red Mexicana de Organizaciones Campesinas Forestales (Mocaf) y Movimiento Agrario Indigena Zapatista (MAIZ). 
el Grupo de Acción contra la Erosión, Tecnología y Concentración (ETC) y el Centro de Estudios para el Cambio en el Campo Mexicano, que participaban en la Red por la Defensa del Maíz (entrevista con integrante de ETC, Ciudad de México, 17 de mayo de 2010). El rechazo de estas organizaciones a asistir todas a un mismo foro se debió en parte a las confrontaciones que habían tenido por la firma del ANC y los pobres resultados que éste había arrojado hasta el momento.

La división en Cancún no fue menor. Si bien algunos líderes de organizaciones campesinas pensaron que el foro separado era una cuestión momentánea, la convivencia con La Vía Campesina y el respaldo del EZLN al Foro Campesino e Indígena permitió a las organizaciones indígenas o que tenían adscripción de bases indígenas amplias repensar la pertinencia de su continuidad en un movimiento campesino que solía tomar decisiones basadas en el consenso de los liderazgos. ${ }^{10}$

\section{La consolidación de la Red en Defensa del Maíz}

En 2005, se decretó la Ley de Bioseguridad de Organismos Genéticamente Modificados y se creó la Comisión Intersecretarial de Bioseguridad de los Organismos Genéticamente Modificados (Cibiogem), dentro de la cual se instalaría un consejo consultivo mixto en el que podrían participar organizaciones de la sociedad civil junto con científicos y empresas, para discutir temas relacionados y elaborar las reglas de operación de los instrumentos derivados de la ley y de la introducción de transgénicos, que a partir de ese momento era posible.

La decisión de participar o no, tanto de organizaciones campesinas como de ONG, estuvo permeada por una serie de consideraciones respecto a lo que implicaría sentarse de nuevo a negociar, o intentar negociar, con instituciones de gobierno la regulación de organismos genéticamente modificados, sobre todo de maíz, cultivo básico en México y de gran simbolismo cultural para los pueblos indígenas de varios países latinoamericanos (Trueba, 2009). La introducción de semilla modificada para siembra, junto con paquetes tecnológicos, es una opción que atenta social, cultural y económicamente contra los sistemas tradicionales de policultivo - la milpa-, pues implica: 1) siembra de semilla homogénea - una o dos variedades-, y 2) uso incremental de herbicidas y fertilizantes que elevan los costos de producción, impiden los cultivos simultáneos y disminuyen de manera gradual la calidad de los suelos.

El análisis de la Red en Defensa del Maíz, espacio que había sido abandonado por las organizaciones campesinas firmantes del ANC, que también organizaron el Foro de los Pueblos en Cancún, fue que asistir constituía validar la introducción de grano transgénico, en tanto que sólo se discutirían los términos de su introducción, no su posibilidad. ${ }^{11}$ Algunas $\mathrm{ONG}^{12}$ decidieron asistir para presentar argumentos de oposición a la liberación de permisos de cultivo de maíz transgénico, lo cual se logró hasta cierto punto. ${ }^{13}$ Sin embargo, para el espacio amplio de la Red, esta participación implicaba validar la introducción de semilla transgénica y condenó la asistencia a las reuniones, lo que marcó una separación

10 La afirmación se hace con base en las entrevistas con miembros de la Red en Defensa del Maíz (Ciudad de México, 17 de mayo de 2010) y representantes de comunidades ñhañús, nahuas y mayas (Guadalajara, Jalisco, 3 de mayo de 2010). Para más detalles sobre las implicaciones de Cancún para la división del movimiento, véase Pardo (2011).

11 Comunicación personal con integrantes del CECCAM (Ciudad de México, 26 de agosto de 2010); Genetic Resources Action International (Grain, Ciudad de México, 11 de agosto de 2010); ETC (Ciudad de México, 17 de mayo de 2010); Colectivo Coa (Guadalajara, Jalisco, 3 de marzo de 2010).

12 Grupo de Estudios Ambientales (GEA), Greenpeace y Semillas de Vida, principalmente.

13 Se consiguió decretar zonas libres de transgénicos basadas en puntos de domesticación de cultivos. 
entre las ONG asistentes y la Red (entrevista con integrante de Semillas de Vida, Ciudad de México, 13 de septiembre de 2010).

Las ONG abandonaron el espacio del Consejo al darse cuenta de que su participación era sólo figurativa para avalar la introducción de transgénicos al campo mexicano (Marielle y Peralta, 2007). La Red en Defensa del Maíz tomó la decisión, en asamblea, de adherirse al movimiento indígena en atención a una invitación recibida durante el Foro Internacional Campesino e Indígena en Cancún, pero también a partir de un análisis de la actualidad del movimiento campesino, ante los sucesos de años anteriores. Pese a que las demandas habían incluido reivindicaciones indígenas y la Red había hecho algunas de corte político, el balance general dejó una sensación de traición por parte de los liderazgos campesinos y del Estado, que además de incumplir los acuerdos pactados, tomaba decisiones adversas a la agricultura campesina (entrevistas con integrantes del Cenami y GEA, Guadalajara, Jalisco, 2 de marzo de 2010; y representantes de pueblos indígenas participantes en la Red y La Vía Campesina, Guadalajara, Jalisco, 3 de marzo de 2010). La decisión de la Red, ante la serie de acuerdos infructuosos y la ola continua de políticas globalizadoras del gobierno, fue convertirse en un movimiento deoposición a la política gubernamental agrícola mediante una resistencia desde el territorio, regido por el CNI, para el cual los intentos de negociación con el gobierno eran tácticas en arenas no aceptables.

Las organizaciones campesinas nacionales amplias del movimiento campesino, que habían cesado su participación en ese espacio, no lo ocuparon más debido a: 1) una agenda acotada a la defensa del maíz nativo, y 2) la decisión de no negociar con el gobierno, lo cual constituía la antítesis de su razón de ser, pues buscaban espacios para la comercialización de sus productos e incidencia en las políticas agrícolas del país.

\section{Campaña Nacional Sin Maíz No Hay País}

En 2007, ante el alza en los precios de la tortilla y la nueva liberación de aranceles para el maíz blanco, algunas organizaciones campesinas que participaron en la Red en un comienzo, pero que la dejaron para impulsar el MECNAM — en particular, el Consejo Nacional de Organizaciones Campesinas (Conoc), la Coordinadora Nacional Plan de Ayala (CNPA), el Barzón Nacional, el Frente Democrático Campesino de Chihuahua y algunas ONG que trabajan temas de alimentación y derechos humanos, como el Instituto de Estudios para el Desarrollo Rural Maya, Semillas de Vida, Greenpeace México y el Centro de Derechos Humanos Fray Francisco de Vitoria-, lanzaron una campaña de carácter informativo y de denuncia llamada "Sin maíz no hay país. ¡Pon a México en tu boca!". Como propuesta de agenda, la convocatoria rescató demandas del MECNAM, como sacar el maíz y el frijol del TLCAN. Otras demandas incluían prohibir la siembra de maíz transgénico, reconocer los derechos de los pueblos originarios y proteger los territorios campesinos, aprobar el derecho constitucional a la alimentación y luchar contra los monopolios del sector agroalimentario (Perelmuter, 2009).

A la convocatoria inicial para la campaña fueron invitadas algunas organizaciones de la Red en Defensa del Maíz, que decidieron no continuar porque "se tenía una agenda predefinida que implicaba el diálogo con [el] gobierno, en puntos que, quedaba claro, no iban a prosperar" (entrevista con integrante del Cenami, Ciudad de México, 29 de junio de 2010). La idea de insistir en puntos que ya habían generado conflicto y en arenas de negociación con el gobierno, a las que la Red había decidido no recurrir, constituyeron los principales motivos de la negativa de la Red.

La Campaña tenía dos arenas de incidencia: la sociedad civil y el Estado, con los objetivos de: 1) difundir entre la sociedad civil los problemas de millones de familias campesinas; 2) lograr la adherencia 
de organizaciones campesinas nacionales para promover la soberanía alimentaria, y 3 ) incidir en políticas públicas en materia agrícola (Cobo, 2014).

La Campaña había previsto un año de actividades; sin embargo, la entrada del maíz y el frijol libres de gravámenes al TLCAN permitió la renovación de votos de unidad entre las organizaciones constituyentes,${ }^{14}$ las cuales decidieron seguir reforzando la difusión y la exigencia de soberanía alimentaria como una demanda hacia el Estado, lo que implicaba la priorización de la producción nacional para el abastecimiento de los mercados internos, así como el apoyo a formas no industrializadas de agricultura, en oposición a los paquetes tecnológicos de uso de semilla e insumos químicos provistos por empresas agrícolas internacionales, cuyos costos financieros y ambientales son elevados.

\section{Identidades y asignación de funciones en los movimientos}

$\mathrm{Al}$ adherirse de forma abierta al movimiento indígena, cuya instancia máxima de deliberación es el CNI, las organizaciones que permanecían en la Red se separaron del movimiento campesino para integrarse al movimiento indígena, lo que implicaba modificar la estructura interna de funcionamiento, las demandas y su relación con el Estado, puesto que, en atención a los lineamientos del CNI, las decisiones de acción y de movimiento corresponden a las asambleas de los pueblos, ejidos o comunidades, no a representaciones o liderazgos personalizados. De esta forma, la figura de organización como asociación por intereses se desdibujó para dar paso a la comunidad como asociación por pertenencia étnica y vecindad geográfica. En la Red, los grupos indígenas colocan su identidad —nahua, triqui, ñhañú, tseltal, etc. - como la fuerza impulsora de su participación. Esto conlleva una normatividad y formas de relacionarse con otros grupos. Cada grupo étnico tiene su cosmovisión particular del maíz, y ceremonias agrícolas y sociales propias, aunque comparten la milpa como forma de cultivo.

La milpa, en la cual el maíz es central, marca los calendarios de la vida comunitaria y familiar, incluyendo las fiestas, así como las dietas y sus variantes anuales (Toledo y Barrera-Bassols, 2008; Bartra y Otero, 2008). El intercambio de semillas y saberes entre campesinos ha contribuido a generar más de 100 variedades de maíz en México. Cada una se adapta al gusto local y a las condiciones ambientales que favorecen su rendimiento.

Los otros actores colectivos que participan en la Red son $\mathrm{ONG}^{15}$ que conciben y han acordado su función como instrumentos de apoyo para las necesidades informativas o técnicas de los actores indígenas. El compromiso de supeditación de su participación a las necesidades de las comunidades indígenas no implica que no tengan otros proyectos. De hecho, la Red es uno más de los espacios en los que se involucran.

Las ONG de la Red, y dentro de ella, definen su identidad en torno a la función de apoyo y refuerzo del proyecto de defensa del maíz en su contexto de cultivo central para México, en el entendido de que su conservación está en manos de quienes lo cultivan y significan: los pueblos indígenas. La Red, como movimiento y parte del CNI, comienza por destacar la marginación de los indígenas como pueblos históricamente ignorados por el gobierno, que reclaman el entendimiento y reconocimiento de sus diferencias culturales, sus especificidades productivas y sus formas tradicionales de gobierno para

14

En ese momento, 20 organizaciones campesinas nacionales. La conformación de la Campaña a lo largo del tiempo puede revisarse a profundidad en Perelmuter (2009); Cobo (2014).

15 La mayoría, de investigación: Colectivo Coa, ETC, CECCAM, Grain. 
realizar un proyecto de soberanía alimentaria que les permita ser autosuficientes, en apego a sus costumbres; además de definir con libertad sus formas de producción y variedades de cultivo.

La Campaña está constituida por ONG y organizaciones campesinas. Éstas exponen los aspectos productivos que aglutinan a sus integrantes en torno a actividades económicas. En tanto que una parte importante de sus demandas se concentra en la modificación de las políticas agrícolas y la apertura de los mercados internos para la producción nacional, necesitan el diálogo con el gobierno. Aunque no todas las organizaciones campesinas producen maíz, todas rechazan los paquetes tecnológicos que otorga el gobierno y que implican el uso de variedades específicas de cultivos y agroquímicos.

Las organizaciones campesinas de la Campaña trabajan en todo el país y tienen oficinas en la Ciudad de México. Sus representantes asisten a las reuniones y pueden tomar decisiones en el momento. Aunque en los núcleos comunitarios de estas organizaciones muchos de los integrantes se identifican como indígenas antes que como campesinos, se participa desde la visión productiva y política que da sentido a la organización amplia. Se busca en la Campaña un espacio de confluencia con otras organizaciones para exigir al gobierno la reivindicación del sector campesino mediante políticas de apoyo a la producción nacional diversificada y al abasto interno, que implica acopio y distribución.

Los otros actores colectivos en la Campaña son ONG. Si bien en su quehacer diario y en sus proyectos no difieren de los grupos que participan en la Red, lo hacen en la concepción de su protagonismo dentro del movimiento. La perspectiva también es distinta a la de las organizaciones campesinas respecto a su participación, impulsada por sus proyectos institucionales como ejes rectores de su quehacer en el movimiento, en el que participan en términos de igualdad de funciones con las otras organizaciones.

\section{Organización social de los movimientos}

Los objetivos del proyecto del CNI son construir la autonomía de los pueblos indígenas en los hechos y garantizar la autodeterminación, ya sea de manera constitucional o en la normatividad que rige a las comunidades (Hernández, 2009: 37). Desde 2002, cuando el CNI reivindicó los Acuerdos en Defensa del Maíz y empezó a participar de manera activa en la Red, los pueblos indígenas están en el centro del proyecto.

A pesar de que asisten más de 15 etnias de las regiones norte, centro, sur, istmo y sureste del país, cada una con su cosmovisión específica que motiva la defensa del maíz nativo, las formas organizativas tradicionales son similares y han permitido establecer una normativa única. Los grupos indígenas participan con comisiones o comisionados definidos por la asamblea comunitaria, a las que regresan con las propuestas del movimiento para ratificarlas e incorporarlas a las actas de gobierno de la comunidad. Las asambleas de la Red se celebran una vez al año y sus acuerdos son ratificados, y en su caso, normativizados en la comunidad.

Las ONG se encargan de tareas logísticas, como gestionar los recursos para la celebración de asambleas o reuniones generales, coordinar actividades, recopilar información, etc. También dan seguimiento a los acuerdos que se toman en la asamblea, como el monitoreo de contaminación transgénica en milpas, pero no tienen un papel resolutivo. Tienen voz en las asambleas, mas no voto. Las ONG de la Red fungen como fuentes de información y vinculación con actores nacionales o internacionales, que pueden aportar nuevos elementos de análisis sobre el impacto de los transgénicos.

La Campaña tiene una estructura menos centralizada para la toma y ejecución de decisiones estratégicas. Por consenso, en las primeras reuniones se acordó no personalizar la representación. Se trabaja como 


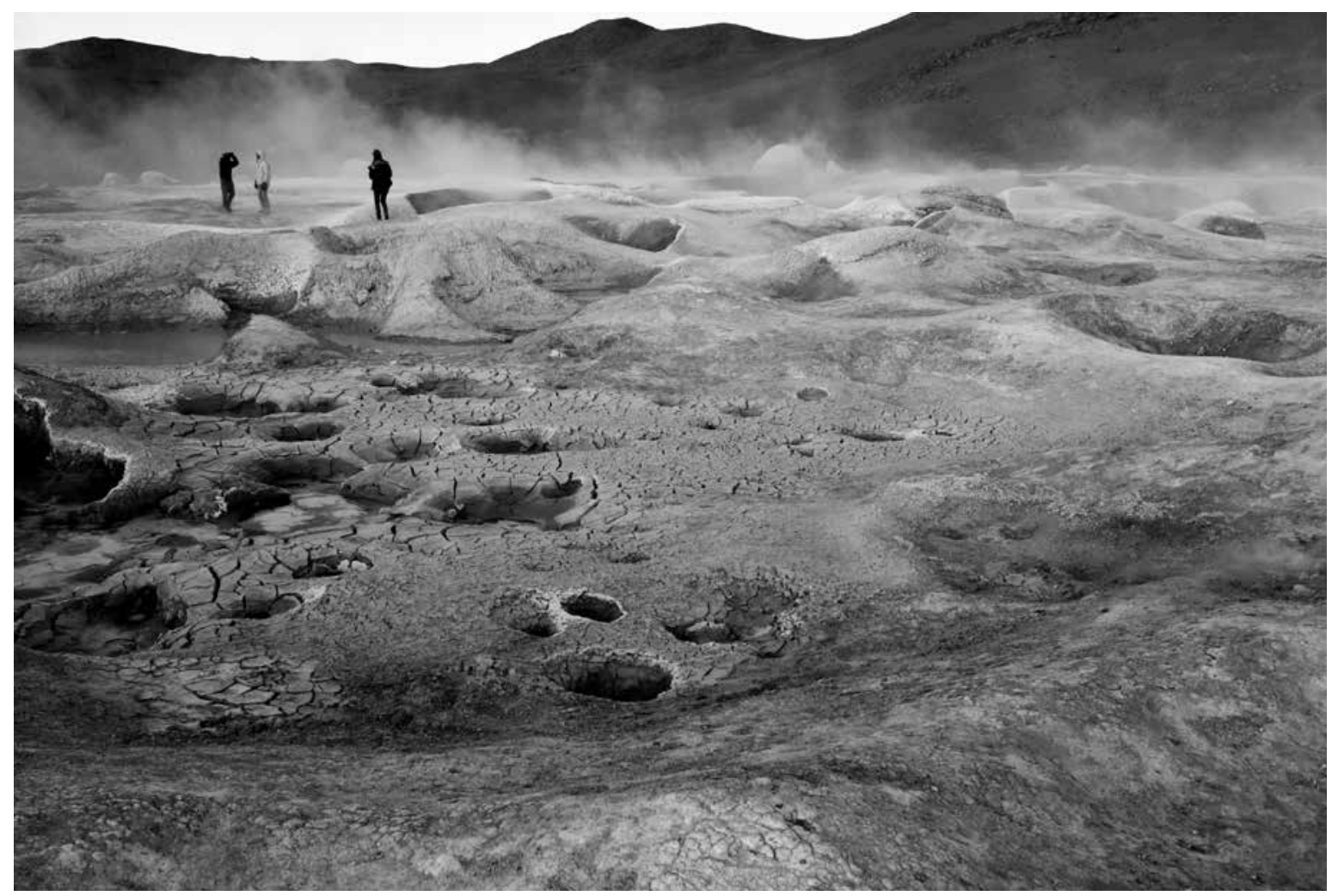

Ricardo Ramirez Arriola/360 Amanecer en el salar de Uyuni. Provincia de Daniel Campos, departamento de Potosí, Bolivia, 2015.

colectivo democrático enfocado en la difusión pública y la incidencia política, en torno a los temas de interés comunes (entrevista con integrante del Instituto Maya de Estudios Rurales, Ciudad de México, 13 de marzo de 2010). Esta decisión obliga a colocar en una instancia colectiva los procesos de deliberación.

Esto llevó a pensar en un equipo coordinador integrado por representantes o delegados de todas las organizaciones que pudieran mantener la continuidad al colocar sus visiones y proyectos institucionales en torno a la soberanía alimentaria como parte de un proyecto amplio, que reflejara la diversidad de actores y proyectos participantes.

La participación de los representantes campesinos no es constante, por ello el equipo denominado "coordinador" tampoco tiene una composición fija: participan quienes asisten, de la misma forma en que se da seguimiento sólo a las acciones impulsadas con constancia, lo que recae casi exclusivamente en las ONG. En este sentido, se emplea el lema "la Campaña es de quien la trabaja". Las reuniones de la Campaña son constantes y en todas se toman decisiones para la acción inmediata.

\section{Decisiones estratégicas}

Las decisiones estratégicas de cada movimiento se vinculan con los actores colectivos que lo conforman, en particular con sus identidades y las funciones acordadas. Cada uno evalúa repertorios y define los que son aceptables para la acción, de acuerdo con la situación que elaboran del problema, es decir, con la delimitación e interpretación 
cultural y contextual que le dan (Benford y Snow, 2000). Reviso a continuación las decisiones estratégicas de ambos movimientos en torno a sus demandas, arenas y tácticas.

\section{Demandas}

Las demandas de la Red han variado con su composición identitaria. En 2002, como resultado del Primer Seminario en Defensa del Maíz, ${ }^{16}$ la Red exigía al gobierno mexicano acciones de política interna entre las que se contaban declarar recurso estratégico al maíz; revisar el capítulo agrícola del TLCAN, las políticas de precios y los subsidios para compensar la pérdida de rentabilidad del maíz; suspender las importaciones de maíz transgénico; limpiar y compensar los espacios contaminados de transgénicos; proteger semillas nativas; respetar la autodeterminación de los pueblos indígenas, y participar en la elaboración de leyes agrícolas y pecuarias. También demandaba que la Organización de las Naciones Unidas para la Alimentación y la Agricultura (FAO, por sus siglas en inglés) reconociera el concepto de soberanía alimentaria, así como una moratoria a la liberación de transgénicos en centros de origen y diversidad de cultivos.

La Red se oponía de manera contundente a la legalización de la siembra de maíz transgénico, pero tenía una diversidad de demandas que implicaban un diálogo con el gobierno, así como exigencias políticas y presupuestales para el campo, en un momento en que su conformación incluía organizaciones campesinas.

La nula respuesta del gobierno, los sucesos posteriores en Cancún, la aprobación y decreto de la Ley de Bioseguridad, y sobre todo, su adscripción al movimiento indígena, sentaron las bases para que la Red endureciera su postura. En 2004, sacó de su agenda las demandas no relacionadas con la introducción de transgénicos. Las organizaciones participantes en la Red decidieron resaltar la presencia indígena y el rechazo al maíz transgénico desde su significado cultural y social.

El matiz de las demandas también se modificó. $\mathrm{Al}$ dejar de exigir de manera directa al gobierno, formularon narrativas que ponían en evidencia la falta de actuación gubernamental y la forma en que las políticas actuales atentaban contra las formas de vida de las comunidades indígenas, con programas que fomentaban el uso de paquetes tecnológicos comprados a las transnacionales agrícolas. Al mismo tiempo, reivindicaron como solución las formas comunitarias de gobierno sobre el territorio y los calendarios y cultivos tradicionales.

Para la Red sólo hay una demanda: "no al maíz transgénico en territorio nacional”. Mientras existan puntos de experimentación o liberación controlada, sólo hay un camino posible de acción: defender de manera integral el territorio, la milpa y la comunidad, al impedir el paso de los paquetes gubernamentales de apoyo que atentan contra estos elementos.

Por su parte, la Campaña decidió desde un primer momento entablar la lucha con una política de incidencia dual: hacia la sociedad civil, dirigida a la gente en su calidad de ciudadana y consumidora, y hacia el gobierno, para impedir o minimizar los impactos negativos de la política agrícola para pequeños productores, incluyendo el rechazo al maíz transgénico.

En la Campaña, las demandas se estructuran en función de una gran diversidad de intereses de trabajo para actores con distintos proyectos. Cada organización en la Campaña enmarca, desde la perspectiva temática e ideológica de su trabajo, el problema de los transgénicos, la falta de políticas de apoyo al campo y los efectos de las políticas de apertura comercial agrícola a grandes transnacionales,

Véase <http://www.ceccam.org.mx/ConclusionesDefensa. $\mathrm{htm}>$. 
que constituyen los tres conflictos alrededor de los cuales se estructuran las demandas y se demanda la soberanía alimentaria.

La estructura social y organizativa de la Campaña no permite apelar a identidades culturales homogéneas. La diversidad de intereses en la Campaña hace que con frecuencia se impulsen demandas en respuesta a los cambios políticos que afectan más. Por ejemplo, durante 2010, se empleó como marco general la crisis alimentaria y el alza de precios de los alimentos. Las demandas destacaron la necesidad de reforzar los apoyos y mercados para la producción campesina, en oposición a los programas Procampo y Proagro. ${ }^{17}$ No obstante, desde 2013 ha sido más importante la lucha contra la liberación de permisos para el cultivo comercial de maíz transgénico.

En términos de demandas, ambos movimientos muestran su oposición a las políticas actuales del gobierno, que promueven paquetes de dependencia tecnológica para la producción y que sólo benefician a los grandes productores, en función del mercado internacional de alimentos.

\section{Arenas}

La Campaña ha optado por la lucha en dos frentes: la vía legal, que implica ocupar la arena política para tratar de incidir en las políticas que afectan al agro; y la opinión pública, en la que procura buscar adherentes en el marco de la sociedad civil, que apoyen la exigencia de un nuevo papel de los campesinos mexicanos en el abastecimiento de los mercados y las despensas nacionales, pero también en sus decisiones de consumo. Las organizaciones de derechos humanos que participan en la Red usan los espacios parlamentarios para impactar en los responsables de tomar las decisiones.

Para la Red, la arena de incidencia se ubica sólo en las comunidades geográficas, con una identidad indígena que les permite reivindicar el maíz como elemento cultural, capaz de impedir el avance de la semilla transgénica en una resistencia desde el territorio, con la siembra de variedades tradicionales con prácticas tradicionales, para crear una barrera a los transgénicos y los paquetes tecnológicos acompañantes. En un segundo plano, para la Red, está la sociedad como una arena que debe tomar conciencia de las implicaciones de permitir la legalización de siembras de transgénicos, cuya simpatía no es determinante.

\section{Tácticas}

Las tácticas de cada movimiento han sido variadas y los procesos internos de decisión no han estado exentos de tensiones. Ambos coinciden en entender como culpable al gobierno, que adopta políticas neoliberales en detrimento de los campesinos y favorece el ensanchamiento de transnacionales agropecuarias para la producción y el abasto del mercado interno. ${ }^{18}$ Sin embargo, estos movimientos se diferencian en las tácticas que adoptan para contender.

Para la Red, la resistencia es la táctica principal, aunque en 2013 tomó la decisión de presentar, junto con la Asamblea Nacional de Afectados Ambientales y La Vía Campesina de Norteamérica, una denuncia frente al Tribunal Permanente de los Pueblos, que amplió la arena de incidencia a la sociedad civil internacional, en la búsqueda de atraer atención a los prejuicios que las políticas de libre comercio tienen hacia las culturas y formas de vida indígenas y campesinas. La Red también promueve las ferias de semillas, espacios regionales en los que convergen

\footnotetext{
17 Programa de atención al campo que sólo se otorga a campesinos que poseen más de cinco hectáreas.

18

Los apoyos al campo no abarcan al grueso de los campesinos pobres, pues en México sólo los pequeños propietarios o las empresas agrícolas cumplen con el requisito de tener cinco hectáreas de cultivo (Fox, 2010).
} 


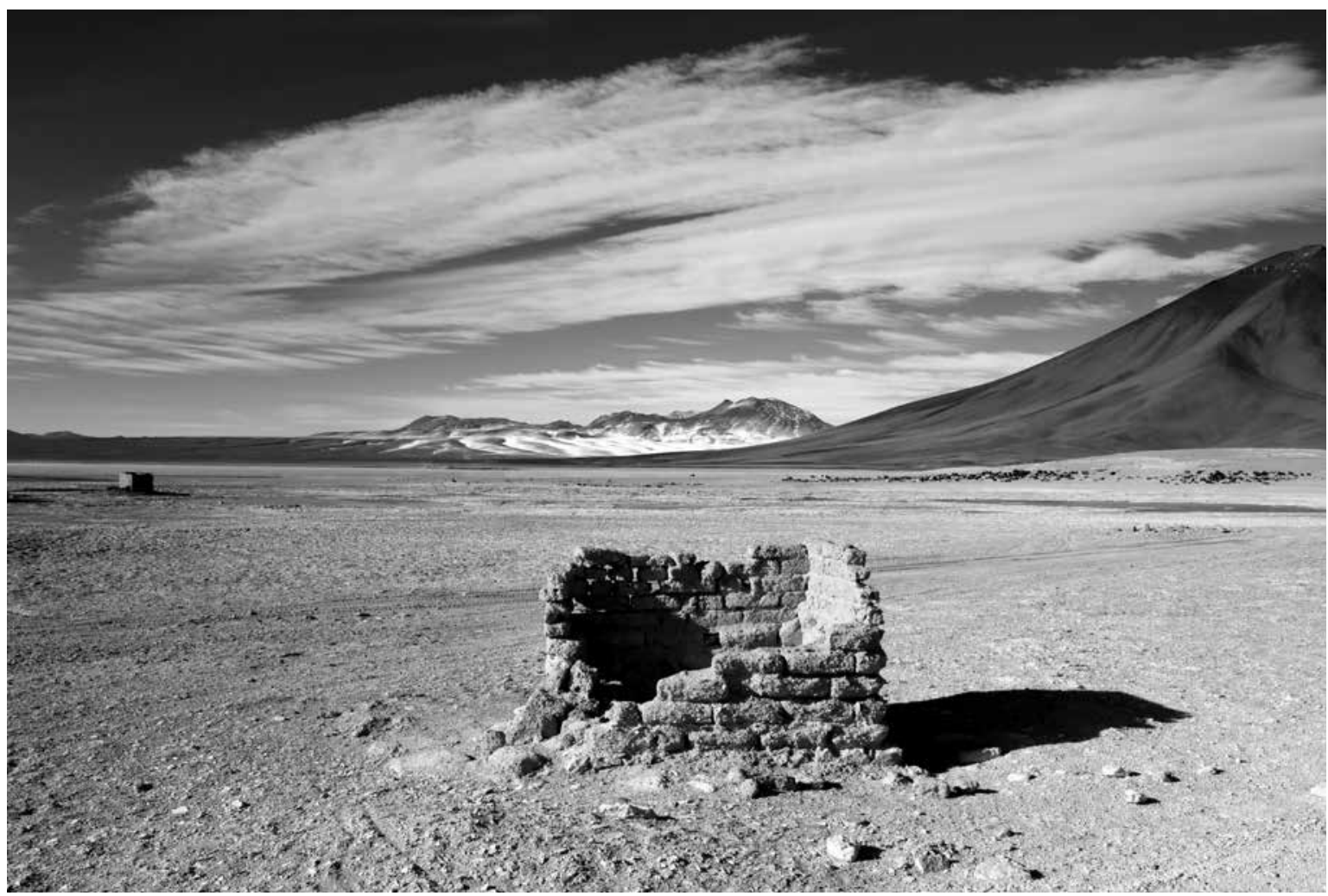

Ricardo Ramírez Arriola/360 Uyuni, el desierto de sal más grande del mundo. Provincia de Daniel Campos, departamento de Potosí, Bolivia, 2015.

campesinos de distintas comunidades para vender o intercambiar semilla de la cosecha para el próximo ciclo de cultivo. Se intercambia también información valiosa para entender las propiedades de cada variedad de maíz y sus mejores formas de cultivo, así se evalúa la pertinencia de mezclar ciertas variedades y se mejora la semilla mediante combinación genética y de saberes.

Para la Red, la defensa del territorio implica la toma de decisiones en asambleas comunitarias. La participación de comunidades enteras es indispensable, pues los acuerdos de mantener un cerco a la introducción de maíz transgénico deben reglamentarse así para ser efectivas.

El trabajo en ejes de la Campaña, la estructura para tomar decisiones y la variedad de organizaciones que la integran han resultado en un repertorio táctico más amplio. La Campaña define sus tácticas como "lucha en diversos frentes", con acciones para lograr adhesiones a las demandas por parte de la sociedad civil. Con este objetivo, se realizan juntas de firmas en eventos públicos o por internet, se llevan a cabo ferias campesinas y urbanas de semillas para visibilizar la diversidad de semillas y cultivos en México, foros, conferencias, mesas redondas y talleres sobre los temas de trabajo de la Campaña a los que invitan académicos y personajes públicos que son parte de la comunidad del movimiento y de la Unión de Científicos Comprometidos con la Sociedad, principalmente. A menudo, se realizan conferencias de prensa ante acciones o comunicaciones del gobierno que conciernen a los temas de la Campaña. Las expresiones artísticas y eventos públicos en los centros urbanos también son una táctica 
empleada para la difusión y adhesión. La Campaña estableció el 29 de septiembre como Día Internacional del Maíz, fecha simbólica de la cosecha de las milpas, día de celebración "diversa como la milpa" para festejar al maíz nativo y a los campesinos que lo siembran en México con eventos en varios estados de la república (Cobo, 2014).

La Campaña también lleva a cabo acciones de exigencia directa al gobierno mediante cabildeo con las cámaras parlamentarias, para ocupar espacios en los Consejos de Desarrollo Rural Sustentable y con presencia en marchas y pliegos petitorios, asistencia a las cámaras parlamentarias y diálogo con personajes políticos para hacer visibles las demandas.

Es importante destacar que en 2014, 53 individuos y 20 organizaciones, tanto de la Red como de la Campaña, decidieron formar una organización sombrilla como representante legal de una demanda colectiva contra el maíz transgénico ante la Corte Suprema de la Nación por los daños que conlleva para la biodiversidad y la salud, entre otros. Esta medida ha tenido que afrontar impugnaciones de Monsanto, Syngenta, Dow, DuPont y las secretarías de Agricultura y Medio Ambiente. El juicio fue declarado procedente en abril de 2015. Entre tanto, se ha declarado una suspensión del cultivo de maíz transgénico como medida precautoria (Ribeiro, 2016). Esta táctica no ha sido suscrita por la Red como colectivo, pero sí por personas y organizaciones que son parte de este movimiento.

\section{Conclusiones}

Los movimientos por la soberanía alimentaria en México contribuyen al debate por la construcción de un nuevo proyecto alimentario en el país. El derrocamiento del régimen alimentario neoliberal es su única apuesta en común, pues sus proyectos de acción para confrontarlo, si bien pueden pensarse como complementarios, no marchan juntos debido a la composición identitaria de cada movimiento, la cual se encuentra fuertemente vinculada a su estructura organizativa para tomar decisiones.

La fragmentación gradual del movimiento campesino derivó en debates que hicieron emerger identidades diferenciadas y motivó a repensar las afinidades y la organización ideal de un movimiento. El perfil identitario y los acuerdos de organización interna de cada movimiento tienen consecuencias que se observan en las decisiones estratégicas y en la imposibilidad de formar un movimiento único.

Para la Red, las comunidades indígenas que participan son las que deben definir y avalar las acciones. Para ellas, el rechazo a negociar con el gobierno se ha convertido en un eje rector. Las tácticas de este movimiento se encaminan a construir una resistencia desde el territorio y buscar la adhesión de más comunidades para establecer un bloqueo al maíz transgénico. La identificación étnica de los actores principales del movimiento y la participación del CNI filtra las opciones del repertorio estratégico.

La Campaña ha sido más enfática y exitosa en denunciar la situación actual del campo, ha enterado a la sociedad civil como ciudadana y consumidora, y ha trascendido el énfasis de la afectación a los integrantes del movimiento hacia la sociedad en general gracias a la conjunción de proyectos múltiples que confluyen y se reflejan como un universo de demandas y opciones para redefinir el papel del campo y los campesinos en el país.

El cuadro 1 muestra las principales diferencias en la configuración interna de cada movimiento y en sus decisiones estratégicas, que reflejan sus acciones e ideales hacia el exterior.

Las formas de enmarcar el problema para hacer un diagnóstico y plantear las posibles soluciones se definen en terrenos distintos en cada movimiento. Para las necesidades productivas de las organizaciones campesinas de la Campaña, es inevitable la lucha en la arena política para buscar de manera constante la negociación, y para que ésta se traduzca 


\begin{tabular}{|c|c|c|}
\hline & Campaña Nacional Sin Maíz No Hay País & Red en Defensa del Maíz \\
\hline Actores principales & $\begin{array}{l}\text { - Organizaciones campesinas de índole } \\
\text { económica } \\
\text { - Organizaciones no gubernamentales } \\
\text { de índole social }\end{array}$ & $\begin{array}{l}\text { Comunidades indígenas de índole étnico- } \\
\text { cultural }\end{array}$ \\
\hline Toma de decisiones & $\begin{array}{l}\text { Coordinación formada por asistentes a } \\
\text { reuniones }\end{array}$ & Asambleas comunitarias \\
\hline Tierra & Recurso productivo de índole social & Territorio \\
\hline Papel de las ONG & $\begin{array}{l}\text { En horizontalidad con actores campesinos } \\
\text { y académicos }\end{array}$ & $\begin{array}{l}\text { Supeditadas a las decisiones de } \\
\text { las comunidades }\end{array}$ \\
\hline $\begin{array}{l}\text { Unidad de análisis } \\
\text { interno }\end{array}$ & Procesos productivos & Procesos culturales \\
\hline Arenas de incidencia & $\begin{array}{l}\text { Sociedad civil - público en general y oNG-, } \\
\text { organizaciones campesinas, Estado en } \\
\text { varios ámbitos y niveles de gobierno }\end{array}$ & $\begin{array}{l}\text { Sociedad civil en el ámbito rural, en } \\
\text { particular comunidades indígenas }\end{array}$ \\
\hline Demandas & $\begin{array}{l}\text { - Modificación de las políticas públicas para } \\
\text { incluir apoyo a los modos de producción } \\
\text { campesinos, diversificados } \\
\text { - Redefinición del papel de los campesinos } \\
\text { en el abasto de mercados internos } \\
\text { agroalimentarios } \\
\text { - Oposición a la siembra de maíz } \\
\text { transgénico }\end{array}$ & $\begin{array}{l}\text { Prohibición de la introducción de maíz } \\
\text { transgénico al país }\end{array}$ \\
\hline Tácticas & $\begin{array}{l}\text { Movilizaciones públicas, eventos sociales } \\
\text { y artísticos de denuncia, publicaciones, } \\
\text { eventos académicos, incidencia en grupos } \\
\text { parlamentarios }\end{array}$ & $\begin{array}{l}\text { Resistencia territorial a la entrada } \\
\text { de paquetes tecnológicos agrícolas }\end{array}$ \\
\hline
\end{tabular}

Fuente: Elaboración propia con base en información de entrevistas.

en políticas para la producción y el comercio de la producción de organizaciones campesinas, lo que no se puede exigir a ninguna otra esfera. La lucha parte de pensar en el avance de las políticas neoliberales como algo inevitable y en las cuales es indispensable incidir.

La Red cuestiona los códigos culturales que sustentan la política agrícola. Busca la creación de un nuevo proyecto de sociedad en el ámbito rural mediante acciones que fortalecen el tejido comunitario y la resistencia ante políticas en cuyo terreno es inútil combatir. La traición del gobierno, que desvirtuó los Acuerdos de San Andrés en la Ley de
Derechos Indígenas, constituyó para el CNI el establecimiento de una moratoria a la negociación con el gobierno, la cual debe ser acatada por cualquiera de sus participantes, incluso la Red.

Las diferencias estratégicas, si bien son producto de estructuras organizativas distintas, evidentes para el observador externo, son el resultado de relaciones sociales que requirieron tiempo para definirse como afinidades o como divergencias entre actores cuya identidad conlleva formas de acción específicas.

La organización interna del movimiento, entendida como la asignación consensuada de funciones o roles, la toma de decisiones, la planeación y el 
flujo de información, es fundamental para conocer el ideal social del movimiento (Chihu, 2000). Las formas organizativas que cada movimiento ha escogido dependen en gran parte del ideal de sociedad de sus miembros (Ganz, 2000).

Cada organización vierte sus ideales de funcionamiento y trascendencia social en la definición de formas operativas. El resultado final, la estructura de comunicaciones, las jerarquías y la función de cada actor colectivo, es producto del consenso generado al plantear e imaginar el problema, sus impactos y soluciones (Benford y Snow 2000; Gamson, 2003), lo cual no puede desligarse de las identidades que lo constituyen y de las formas en las que se organiza para funcionar. $\mathbf{D}$

\section{Bibliografía}

Bartra, Armando y Gerardo Otero, 2008, "Movimientos indígenas campesinos en México: la lucha por la tierra, la autonomía y la democracia”, en Sam Moyo y Paris Yeros (eds.), El resurgimiento de movimientos rurales en África, Asia y América Latina, Consejo Latinoamericano de Ciencias Sociales, Buenos Aires.

Benford, Robert D. y David Snow, 2000, "Framing Processes and Social Movements: An Overview and Assessment", en Annual Review of Sociology, vol. 26, pp. 611-639.

Canto, Manuel, 2002, De lo cívico a lo público: una discusión de las organizaciones civiles, Red Mexicana de Investigadores sobre Organizaciones Civiles/Centro de Estudios Sociales y Culturales Antonio de Montesinos, México.

Cardoso de Oliveira, Roberto, 2007, Etnicidad y estructura social, Centro de Investigación y Estudios Superiores en Antropología Social/ Universidad Autónoma Metropolitana-Iztapalapa/Universidad Iberoamericana, México.

Carrillo Trueba, César, 2008, “El origen del maíz. Naturaleza y cultura en Mesoamérica”, en Ciencias, núms. 92-93, pp. 4-13.

Chihu Amparán, Aquiles, 2000, "Melucci: la teoría de la acción colectiva”, en Argumentos, núm. 37, pp. 79-92.

Cobo, María del Rosario, 2014, La Campaña Nacional Sin Maíz No Hay País: alcances y desafíos de una red de redes en movimiento, tesis de doctorado en desarrollo rural, Universidad Autónoma Metropolitana-Xochimilco, México. Disponible en línea: <http://bocadepolen. org/web/wp-content/uploads/2014/04/Tesis-de-la-Campa\%C3\%Bla-Chaca.pdf>.

Cohen, Jean y Andrew Arato, 2001, Sociedad civil y teoría política, Fondo de Cultura Económica, México.

Concheiro Bórquez, Luciano, María Tarrío y Sergio Grajales, 2007, "El TLCAN al filo de la navaja: notas para una propuesta de renegociación", en Liminar. Estudios Sociales y Humanísticos, vol. 5, núm. 2, pp. 108-128.

Cueva, Héctor de la, 2003, "La batalla de Cancún: balance de una victoria”, en Observatorio Social de América Latina, vol. 4, núm. 11, pp. 281-290.

Edwards, Bob y John D. McCarthy, 2004, "Strategy Matters: The Contingent Value of Social Capital in the Survival of Local Social Movement Organizations”, en Social Forces, vol. 83, núm. 2, pp. 621-651.

Fox, Johnatan, 2010, "Siete mitos sobre los subsidios agrícolas mexicanos", ponencia presentada en el Seminario Internacional "Transparencia y rendición de cuentas en los subsidios agrícolas: políticas públicas y modelos de desarrollo rural”, Facultad de Economía-Universidad Nacional Autónoma de México, México, 1 de septiembre. Disponible en línea: <http://subsidiosalcampo.org.mx/wp-includes/textos_ pdf/otros/ponenciajf.pdf>. Consultado el 20 de julio de 2017.

Freeman, Jo, 1999, "A Model for Analyzing the Strategic Options for Social Movement Organizations", en Jo Freeman y Victoria Johnson (eds.), Waves of Protest: Social Movements Since the Sixties, Rowman and Littlefield, Lanham, pp. 241-265.

Gamson, William, 2003, "Bystanders, Public Opinion and the Media”, en David A. Snow, Sarah A. Soule y Hanspeter Kriesi (eds.), The Blackwell Companion to Social Movements, Blackwell, Londres, pp. 242-261.

Ganz, Marshall, 2000, "Resources and Resourcefulness: Strategic Capacity in the Unionization of California Agriculture, 1959-1966", en American Journal of Sociology, vol. 105, núm. 4, pp. 1003-1062.

Grammont, Hubert de, Horacio Mackinlay y Richard Stoller, 2009, "Campesino and Indigenous Social Organizations Facing Democratic Transition in Mexico, 1938-2006”, en Latin American Perspectives, vol. 36, núm. 4, pp. 21-40. 
Hernández Navarro, Luis, 2009, "Movimiento indígena: autonomía y representación política”, en Giovanna Gasparello y Jaime Quintana (coords.), Otras geografías. Experiencias de autonomías indígenas en México, Universidad Autónoma Metropolitana-Iztapalapa, México.

Johnson, Erica y Aseem Prakash, 2007, “NGo Research Program: A Collective Action Perspective”, en Policy Sciences, vol. 3, núm. 40, pp. 221-240.

Kane, Anne, 1997, "Theorizing Meaning Construction in Social Movements: Symbolic Structures and Interpretation during the Irish Land War, 1879-1882”, en Sociological Theory, vol. 15, núm. 2, pp. 249-276.

Kokogiannis, Kostas, 2010, "Researching the Emotions in Social Movements: Do the Emotional Experiences Amplify the Action for Change?", ponencia presentada en la conferencia "Between Local and Global: Adult Learning and Development”, European Society for Research on the Education of Adults, Universidad de Sevilla, Sevilla, 20 a 22 de mayo.

Kurtz, Sharon, 2002, Workplace Justice: Organizing Multi-Identity Movements, University of Minnesota Press, Minneapolis.

Lehman, Frederick, 1967, "Ethnic Categories in Burma and the Theory of Social Systems”, en Peter Kunstadter (ed.), Southeast Asian Tribes. Minorities and Nations, vol. 1, Princeton University Press, Nueva Jersey, pp. 93-124.

Lofland, John, 1996, Social Movement Organizations. Guide to Research Insurgent Realities, Aldine de Gruytier, Nueva York.

Mackinlay, Horacio y Gerardo Otero, 2004, "State Corporatism and Peasant Organizations: Toward New Institutional Arrangements", en Gerardo Otero (ed.), Mexico in Transition: Neoliberal Globalism, the State and Civil Society, Zed, Londres, pp. 72-88.

McMichael, Philip, 2006. "Peasant Prospects in the Neoliberalism Age", en New Political Economy, vol. 11, núm. 3, pp. 407-418.

Marielle, Catherine y Lizy Peralta, 2007, La contaminación transgénica del maíz en México. Luchas civiles en defensa del maíz y la soberanía alimentaria, Grupo de Estudios Ambientales, México.

Melucci, Alberto, 2002, Acción colectiva, vida cotidiana y democracia, El Colegio de México, México.

Meyer, David y Suzanne Staggenborg, 2007, "Thinking About Strategy", ponencia presentada en el Collective Behavior/Social Movement Section's Workshop: "Movement Cultures, Strategies, and Outcomes”, American Sociological Association, Hofstra University, Nueva York, 9 y 10 de agosto.

Nyéléni Newsletter, 2007, “Declaración de Nyéléni”, Foro para la Soberanía Alimentaria, Nyéléni, Selingue, 27 de febrero. Disponible en línea: <https://nyeleni.org/spip.php?article291>.

Pardo Núñez, Joaliné, 2011, Identidades, trayectorias y organización en las decisiones estratégicas de un movimiento: la lucha por la soberanía alimentaria en México, tesis de doctorado en investigación en ciencias sociales, Facultad Latinoamericana de Ciencias Sociales, México.

Perelmuter, Tamara, 2009, "Sin maíz no hay país. Análisis de la campaña mexicana", ponencia presentada en el XXVII Congreso de la Asociación Latinoamericana de Sociología, Universidad de Buenos Aires, Buenos Aires. Disponible en línea: <http://www.aacademica. org/000-062/2203.pdf>.

Pleyers, Geoffrey, 2004, "Social Forums as an Ideal Model of Convergence", en International Social Science Journal, vol. 56, núm. 182, pp. 507-517.

__ 2010, "El altermundismo en México. Actores, culturas políticas y prácticas contra el neoliberalismo”, en llán Bizberg y Francisco Zapata (coords.), Los grandes problemas de México, vol. IV: Movimientos sociales, El Colegio de México, México, pp. $361-395$.

Polletta, Francesca y James Jasper, 2001, "Collective Identity and Social Movements”, en Annual Review of Sociology, núm. 27, pp. 283-305.

Quist, David e Ignacio Chapela, 2002, “Transgenic DNA Introgressed into traditional Maize Landraces in Oaxaca, Mexico”, en Nature, vol. 414, núm. 29, pp. 541-543.

Red en Defensa del Maíz, 2003. "Conclusiones del Segundo Seminario 'En defensa del maíz”. Disponible en línea: <http://redendefensadelmaiz.net/conclusiones-del-segundo-seminario-en-defensa-del-maiz/\#\&panel1-7>. Consultado el 19 de junio de 2017.

Ribeiro, Silvia, 2016, “En defensa del maíz, otra vez”, en La Jornada, 14 de mayo.

Rubio, Blanca, 2003, Explotados y excluidos. Los campesinos latinoamericanos en la fase agroexportadora neoliberal, Plaza y Valdés, México. 2006, “Territorio y globalización en México. ¿Un nuevo paradigma rural?”, en Comercio Exterior, vol. 56, núm. 12, pp. 1047-1054.

Sánchez Albarrán, Armando, 2004, "Del movimiento iEl campo no aguanta más! a las movilizaciones sociales en la cumbre de la omc en Cancún. Dependencia o soberanía alimentaria: ésa es la cuestión... agraria”, en El Cotidiano, vol. 19, núm. 124, pp. 41-56.

Serna, Eva, Alejandra Valero y Lucio Díaz, 2010, “De sobrevivientes y guardianes. Luchas campesinas en México”, en Rebeldía, vol. 3, núm. 68, pp. 24-36.

Smithey, Lee Alan, 2002, Strategic Collective Action and Collective Identity Reconstruction: Parading Disputes and Two Northern Ireland Towns, tesis de doctorado en sociología, University of Texas at Austin, Austin. 
Snow, David y Robert Benford, 1988, "Ideology, Frame Resonance and Participant Mobilization”, en Internationa/ Socia/ Movement Research, núm. 1, pp. 197-217.

Staggenborg, Susan, 1998, "Social Movement Communities and Cycles of Protest: The Emergence and Maintenance of a Local Women's Movement", en Social Problems, vol. 45, núm. 2, pp. 180-204.

Suárez Carrera, Víctor, 2005, ¿Tiene futuro la agricultura campesina en México?, Grupo Parlamentario del Partido de la Revolución Democrática, Cámara de Diputados, LIX Legislatura, México.

—_ 2008, Políticas públicas para la agricultura mexicana con base en el consenso y la certidumbre: el caso de la Ley de Planeación para la Soberanía y la Seguridad Agroalimentaria y Nutricional, tesis de ingeniería en agronomía con especialidad en economía agrícola, Universidad Autónoma Chapingo, Chapingo.

Toledo, Víctor y Narciso Barrera-Bassols, 2008, La memoria biocultural, Icaria, Barcelona.

Trueba Carrillo, César, 2009. "El origen del maíz, naturaleza y cultura en Mesoamérica”, en Ciencias, núms. 92-93, pp. 4-13.

Vía Campesina, La, 2016, "Declaración de La Vía Campesina sobre comercio, mercados y desarrollo”. Disponible en línea: <https://viacampesina.org/es/index.php/temas-principales-mainmenu-27/soberanalimentary-comercio-mainmenu-38/2734-declaracion-dela-via-campesina-sobre-comercio-mercados-y-desarrollo>. Consultado el 13 de septiembre de 2016.

\section{Entrevistas}

Integrantes del Centro Nacional de Ayuda a las Misiones Indígenas (Cenami) y el Grupo de Estudios Ambientales (GEA), Guadalajara, Jalisco, 2 de marzo de 2010 .

Representantes de pueblos indígenas participantes en la Red en Defensa del Maíz y La Vía Campesina, Guadalajara, Jalisco, 3 de marzo de 2010.

Representantes de comunidades ñhañús, nahuas y mayas, Guadalajara, Jalisco, 3 de marzo de 2010.

Integrante del Instituto Maya de Estudios Rurales, Ciudad de México, 13 de marzo de 2010.

Integrante del Grupo de Acción contra la Erosión, Tecnología y Concentración (ETC), Ciudad de México, 17 de mayo de 2010.

Miembros de la Red en Defensa del Maíz, Ciudad de México, 17 de mayo de 2010.

Integrantes del Centro Nacional de Ayuda a las Misiones Indígenas (Cenami), Ciudad de México, 29 de junio de 2010.

Integrantes de la Coordinadora Nacional de Organizaciones Cafetaleras (CNOC), Ciudad de México, agosto de 2010.

Integrante del Centro de Estudios para el Cambio en el Campo Mexicano (Ceccam), Ciudad de México, 26 de agosto de 2010.

Integrante de Semillas de Vida, Ciudad de México, 13 de septiembre de 2010. 